\title{
A Downloadable Three-Dimensional Virtual Model of the Visible Ear
}

\author{
Haobing Wang ${ }^{\text {a Saumil N. Merchant }}{ }^{\mathrm{a}-\mathrm{c}}$ Mads S. Sorensen ${ }^{\mathrm{d}}$ \\ a Eaton-Peabody Laboratory of Auditory Physiology, Massachusetts Eye \& Ear Infirmary, \\ ${ }^{b}$ Department of Otology \& Laryngology, Harvard Medical School, Boston, Mass., and \\ 'Harvard-MIT Division of Health Science \& Technology, Cambridge, Mass., USA; \\ ${ }^{\mathrm{d}}$ Department of Otolaryngology Head \& Neck Surgery, Rigshospitalet, University of Copenhagen, Denmark
}

\section{Key Words}

3-D reconstruction $\cdot$ Human temporal bone $\cdot$

Surface rendering

\begin{abstract}
Purpose: To develop a three-dimensional (3-D) virtual model of a human temporal bone and surrounding structures. Methods: A fresh-frozen human temporal bone was serially sectioned and digital images of the surface of the tissue block were recorded (the 'Visible Ear'). The image stack was resampled at a final resolution of $50 \times 50 \times 50 / 100 \mu \mathrm{m} /$ voxel, registered in custom software and segmented in PhotoShop ${ }^{\circledR}$ 7.0. The segmented image layers were imported into Amira ${ }^{\circledR} 3.1$ to generate smooth polygonal surface models. Results: The 3-D virtual model presents the structures of the middle, inner and outer ears in their surgically relevant surroundings. It is packaged within a cross-platform freeware, which allows for full rotation, visibility and transparency control, as well as the ability to slice the 3-D model open at any section. The appropriate raw image can be superimposed on the cleavage plane. The model can be downloadedathttps://research.meei.harvard.edu/Otopathology/ 3dmodels/

Copyright $\odot 2007$ S. Karger AG, Basel
\end{abstract}

\section{Introduction}

The three-dimensional (3-D) anatomy of the human temporal bone can be challenging for students in the basic science or medical disciplines to grasp, because of a large number of complex structures contained within a small space. Knowledge of anatomy of the temporal bone is also critical for the training and planning of otologic surgery. Computer-based representation of 3-D anatomic data has become a useful tool for the study of the temporal bone and for surgical training as a result of recent developments in processing power of modern computers along with 3-D imaging and computer graphics technology. Several investigators have developed 3-D wireframe [1-3] or surface models of the human and animal inner ear using histological [4-9], radiological $[10,11]$ or microscopic images $[12,13]$. Although quite useful, many of these models contain a limited set of anatomical structures, which can be visualized as 2-D images or 3-D movies, but these models lack the capacity for user interaction.

Researchers at the Massachusetts Eye and Ear Infirmary (MEEI) recently developed a 'Teaching Model' of the human temporal bone based on archival, serial, histological sections of the right ear of a 14-year-old male [14]. This model is a set of smooth surface renderings of structures of interest in the external, middle and inner ears. The model incorporates a free, downloadable software package that enables user interactions such as rota-

Mads S. Sorensen, MD

Department of Otolaryngology Head and Neck Surgery F 2074, Rigshospitalet

DK-2100 Copenhagen (Denmark)

Tel. +45 3545 8786, Fax +45 35452690

E-Mail ms.sorensen@dadlnet.dk 
Fig. 1. A photographic image of the surface of the tissue block during cryosectioning of the Visible Ear.

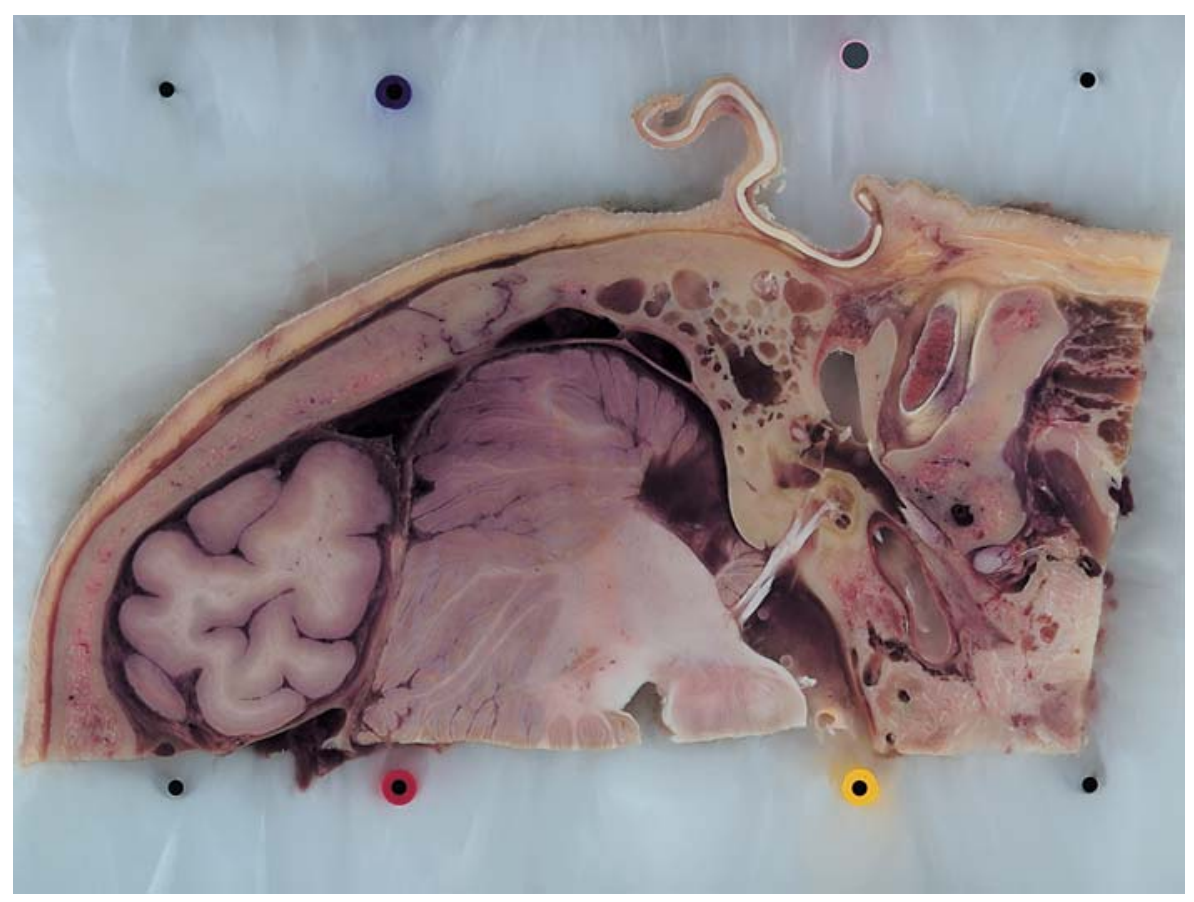

tion, zoom, and control of transparency of each anatomical structure. Although this model contains all major structures of the human temporal bone, has relatively high resolution, and is user interactive, it has certain limitations. For example, the tegmen is missing, along with a part of the superior semicircular canal. The model also does not contain the most posterior part of the mastoid or the structures immediately adjacent to the temporal bone.

Researchers at the University of Copenhagen and Rigshospitalet, Denmark, have published a digital freeware image library named the 'Visible Ear', using a freshfrozen human temporal bone serially sectioned at $25 \mu \mathrm{m}$ with digital images of the block surface recorded at intervals of 50-100 $\mu \mathrm{m}$ [15]. This Visible Ear contains a comprehensive data set of images of the human ear and its surgically relevant surroundings. The use of images acquired from the surface of the block prevented distortions derived from mounting of individual histological sections. The horizontal images can be viewed as a 2-D fly-through movie and the entire volume can be sliced in three perpendicular planes with a low-resolution thumbnail 2-/3-D custom viewer. However, the Visible Ear does not contain a true 3-D reconstruction or capacity for user interaction.

It is apparent that the MEEI Teaching Model and the Visible Ear have complementary strengths and weak- nesses. Therefore, we decided to combine the advantages of both models and generate $3-\mathrm{D}$ reconstructions from the Visible Ear library using the technology of the MEEI Teaching Model.

\section{Materials and Methods}

Cryosectioning, Digitization and Segmentation of Images

The Visible Ear is based on a fresh-frozen human temporal bone from an 85-year-old female with no history of previous ear disease. The bone, which had been donated to the Department of Anatomy, University of Copenhagen, was cryosectioned serially in the axial plane at a thickness of $25 \mu \mathrm{m}$, and digital images of the $12 \times 20 \mathrm{~cm}$ block surface were recorded at 50 - to $100-\mu \mathrm{m}$ increments with a LightPhase ${ }^{\mathrm{TM}}$ (www.phaseone.com) single-shot camera back attachment on a Hasselblad 553 ELX camera (www. hasselblad.com) as previously described [15] (fig. 1). A total of 605 RGB 24-bit images of 3,056 $\times 2,032$ pixels each were brought into registration with custom-made software by the application of a 'least-square best-fit' algorithm of the vertical fiducial markers embedded inside the tissue block, cropped to display a constant area of $15.4 \times 9.7 \mathrm{~cm}$, and resampled to 3,078 $\times 1,942$ pixels at a final resolution of $50 \mu \mathrm{m} /$ pixel.

The images were imported into Adobe Photoshop ${ }^{\circledR} 7.0$ (Adobe Systems Inc., San Jose, Calif., USA) and anatomical structures of interest were segmented manually into 28 different PSD (PhotoShop Document) image layers. Semiautomated selection tools such as 'magic wand' and 'magnetic lasso' were used to speed up the process whenever possible. Precision was enhanced by the use of a Wacom PL-400 ${ }^{\mathrm{TM}}$ digitizer (www.wacom.com), an interactive 
display that allows the user to trace any outline with a pen-shaped pointing device directly on an LCD (liquid crystal display) screen.

\section{Surface Reconstruction, Simplification and Smoothing}

The segmented image layers were imported into Amira ${ }^{\circledR} 3.1$ (Mercury Computer System, San Diego, Calif., USA) to generate 3-D polygonal models, similar to the process used in the MEEI Teaching Model [14]. Since the images were taken directly from the surface of the tissue block, distortions were reduced to a minimum. No interpolation was necessary, and only a minimum amount of smoothing was applied to the surface models. Each anatomical structure was then assigned a color, according to certain rules of convention that we adopted. For example, nerves were given different shades of yellow, arteries red, and veins different shades of blue, etc.

\section{3-D Surface Viewer}

Amira ${ }^{\circledR}$ provides a user-friendly and powerful software environment for creation and representation of 3-D models, however it is relatively expensive, which renders it impractical for wide distribution for teaching purposes. Students and residents in otolaryngology would be able to better appreciate the anatomy and 3-D spatial relationships if they could freely manipulate the 3-D models on their own computers without time or space restraints. Due to these considerations, we developed a standalone, down-loadable, and cross-platform software package, the 3-D Surface Viewer. This software was developed in $\mathrm{C}++$ using OpenGL technology (www.OpenGL.org) and FLTK (Fast Lightweight Toolkit, an open source toolkit for GUI development. www.FLTK.org). Cross-platform compilation was made for Windows XP/2000, Mac OS X, and Linux Red Hat 9. We incorporated the 3-D Virtual Model of the Visible Ear into the 3-D Surface Viewer, and the whole package can be downloaded from ourwebsite athttps://research.meei.harvard.edu/Otopathology/ 3dmodels/

\section{Results}

The 3-D Virtual Model of the Visible Ear is a high-fidelity, anatomically realistic human ear in its surgically relevant surroundings. The model contains 26 anatomical structures organized in six groups: Skin, Soft Tissue \& Bone; Middle Ear; Inner Ear; Cranial Nerves; Major Blood Vessels, and Cranial Cavity. The Skin, Soft Tissue \& Bone group includes skin \& subcutaneous tissue, auricular cartilage, and bone. The Middle Ear group includes the tympanic membrane, malleus, incus, stapes, stapedius muscle, tensor tympani muscle, ossicular ligaments, eustachian tube cartilage, and mastoid air cells. The Inner Ear group includes the cochlea and vestibular sense organs. The Cranial Nerves group includes the lower eight cranial nerves: trigeminal, abducens, facial, cochleovestibular, glossopharyngeal, vagus, spinal accessory, and hypoglossal. The Major Blood Vessels group includes the internal carotid artery, the vertebral artery and branches, and the venous sinuses plus jugular vein. Finally, the Cranial Cavity group includes the dura and the central nervous system - cerebrum, brainstem, cerebellum and cerebrospinal fluid.

The Visible Ear model is packaged with the freeware 3-D Surface Viewer, which provides a user-friendly graphical interface to display and manipulate the 3-D surface models created in Amira ${ }^{\circledR}$ (fig. 2). In the 3-D Surface Viewer, users can rotate and zoom the 3-D models in real time using simple mouse controls. The visibility of each individual anatomical structure can be controlled and the degree of transparency can be adjusted. The 3-D models can be also clipped (sliced open) using the 2-D images, and the 2-D raw images can be superimposed onto the 3-D models at the level of the cleavage plane (fig. 3). The raw stack of images can be re-sectioned in three different orthogonal planes, and users can step through the images in different sections.

\section{Discussion}

The 3-D virtual model of the Visible Ear delivers realistic, interactive anatomic information about the middle, inner and outer ears in their surgically relevant surroundings. It is a useful teaching tool for relating 2-D morphology from sections to the complex 3-D anatomy, as well as for surgical training. The use of photographic images taken from the surface of the tissue block prevents the possible distortions that can occur during mounting of individual histological sections. This method assures the final 3-D model to be of high fidelity and anatomically realistic. The photographic images of the tissue block also retain the true color of the anatomical structures, which makes this data set a good candidate for realistic-looking volume rendering and surgical simulation $[16,17]$. However, the photographic images lack those details in the histological sections which are seen due to staining. Therefore, there are limitations in terms of the level of detail that can be ascertained from 3-D reconstructions made from surface photographic images. For example, it is difficult to segment small structures in the labyrinth, such as the basilar membrane or the ductus reuniens. The existing MEEI Teaching Model shows structures such as the basilar membrane, but is based on histological sections, and therefore is compromised to a small degree by distortions due to histological preparation. With the recent development of non-rigid algorithms to register images, it may be possible in the 
Fig. 2. A screen shot of the virtual model of the Visible Ear displayed in the 3-D Surface Viewer. The dura and CNS have been turned off, and the bone set to be half transparent.
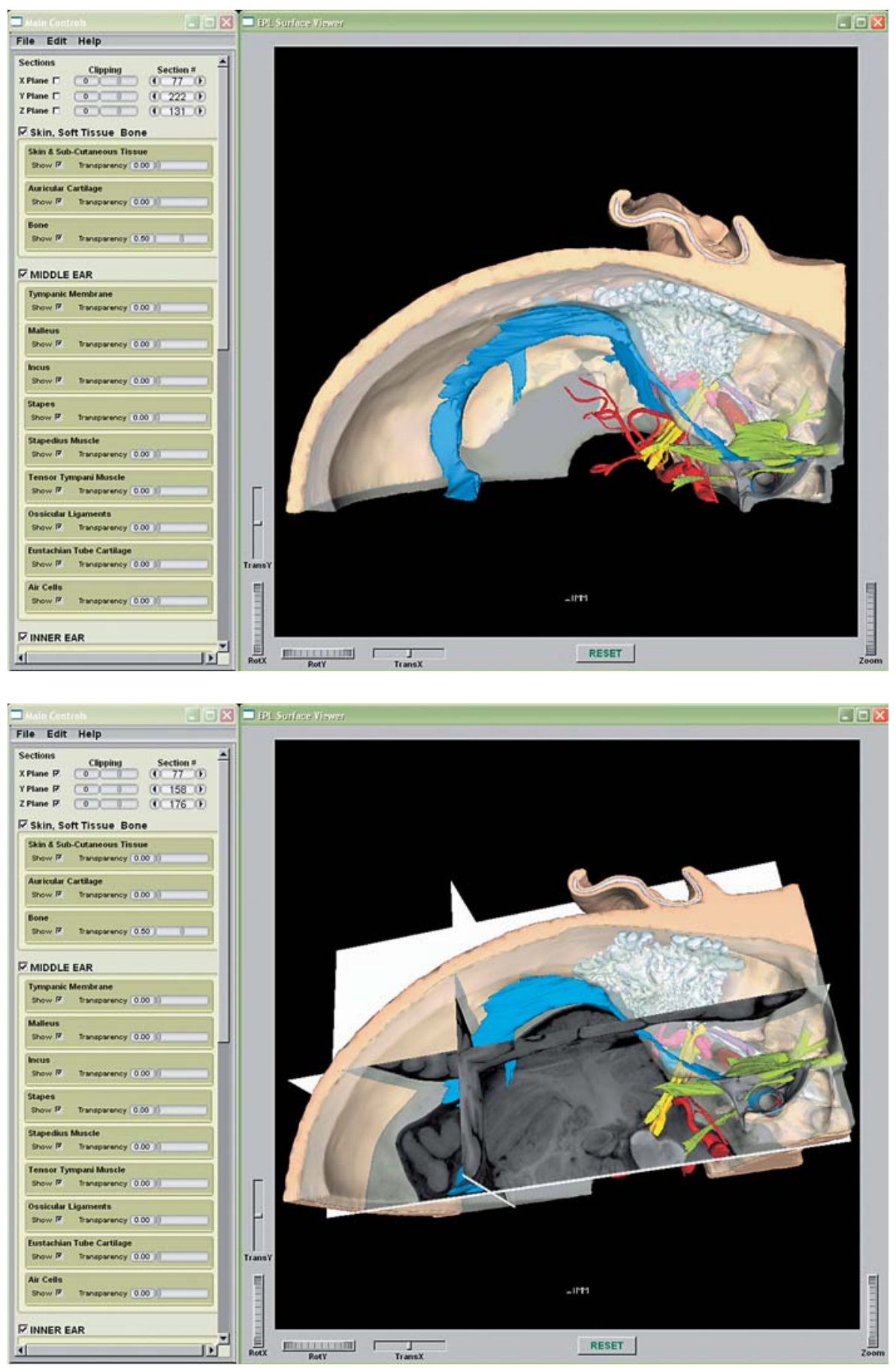

Fig. 3. A screen shot of the virtual model of the Visible Ear displayed in the 3-D Surface Viewer, superimposed with three 2-D images in three orthogonal planes.

\section{Acknowledgement}

This project was supported by a core grant from the NIDCD (P30 DC05209), by a NIH grant (U24 DC008559) and by Mr. Lakshmi Mittal. tailed, yet distortion-free 3-D reconstructions of very small anatomical structures. 


\section{References}

1 Takagi A, Sando I: Computer-aided threedimensional reconstruction and measurement of the vestibular end-organs. Otolaryngol Head Neck Surg 1988;98:195-202.

$\checkmark 2$ Takagi A, Sando I, Takahashi H: Computeraided three-dimensional reconstruction and measurement of semicircular canals and their cristae in man. Acta Otolaryngol 1989; 107:362-365

$\checkmark 3$ Nakashima S, Sando I, Tkahashi H, Fujita S: Computer-aided 3-D reconstruction and measurement of the facial canal and facial nerve. I. Cross-sectional area and diameter: preliminary report. Laryngoscope 1993;103: 1150-1156.

4 Harada T, Ishii S, Tayama N: Three-dimensional reconstruction of the temporal bone from histologic sections. Arch Otolaryngol Head Neck Surg 1988;114:1139-1142.

5 Green JD Jr, Marion MS, Erickson BJ, Robb RA, Hinojosa R: Three-dimensional reconstruction of the temporal bone. Laryngoscope 1990;100:1-4.

6 Mason TP, Applebaum EL, Rasmussen M, Millman A, Evenhouse R, Panko W: Virtual temporal bone: creation and application of a new computer-based teaching tool. Otolaryngol Head Neck Surg 2000;122:168-173.
7 Arnold WH, Lang T: Development of the membranous labyrinth of human embryos and fetuses using computer aided 3-D reconstruction. Ann Anat 2001;183:61-66.

8 Rother T, Schrock-Pauli C, Karmody CS, Bachor E: 3-D reconstruction of the vestibular endorgans in pediatric temporal bones. Hear Res 2003;185:22-34.

-9 Qiu MG, Zhang SX, Liu ZJ, Tan LW, Wang YS, Deng JH, Tang ZS: Plastination and computerized 3-D reconstruction of the temporal bone. Clin Anat 2003;16:300-303.

10 Morra A, Tirelli G, Rimondini V, Cioffi V, Russolo M, Giacomarra V, Pozzi-Mucelli R: Usefulness of virtual endoscopic three-dimensional reconstructions of the middle ear. Acta Otolaryngol 2002;122:382-385.

11 Vrabec JT, Champion SW, Gomez JD, Johnson RF Jr, Chaljub G: 3-D CT imaging method for measuring temporal bone aeration. Acta Otolaryngol 2002;122:831-835.

12 Santi P, Blair A, Pham V, Voie A: The Mouse Cochlea Database: High-Resolution 3-D Reconstructions of the Mouse Cochlea. 27th Midwinter Meeting of the Association for Research in Otolaryngology, Daytona Beach, Fla., February 21-26, 2004
13 Hardie NA, MacDonald G, Rubel EW: A new method for imaging and $3-D$ reconstruction of mammalian cochlea by fluorescent confocal microscopy. Brain Res 2004;1000:200210 .

14 Wang H, Northrop C, Liberman MC, Merchant SM: 3-D Virtual model of the human temporal bone: a stand-alone down-loadable teaching tool. Otol Neurotol 2006;27:452457.

15 Sorensen MS, Dobrzeniecki AB, Larsen P, Frisch T, Sporring J, Darvann TA: The visible ear: a digital image library of the temporal bone. ORL 2002;64:378-381 (including CD, ISBN 3-8055-7534-3).

16 Wiet GJ, Stredney D, Sessanna D, Bryan JA, Welling DB, Schmalbrock P: Virtual temporal bone dissection: an interactive surgical simulator. Otolaryngol Head Neck Surg 2002;127:79-83.

17 Wiet GJ, Schmalbrock P, Powell K, Stredney D: Use of ultra-high-resolution data for temporal bone dissection simulation. Otolaryngol Head Neck Surg 2005;133:911-915. 